Cite this: Org. Biomol. Chem., 2013, 11, 3756

Received 31st January 2013,

Accepted 24th April 2013

DOI: $10.1039 / c 3 o b 40213 d$

www.rsc.org/obc

\section{Manipulating non-innocent $\pi$-spacers: the challenges of using 2,6-disubstituted BODIPY cores within donor-acceptor light-harvesting motifs $\dagger$}

\author{
Catherine Bonnier, Devin D. Machin, Omar Abdi and Bryan D. Koivisto* \\ The syntheses and physicochemical properties for a series of 2,6-disubstituted-4,4-difluoro-4-bora-3a,4a- \\ diaza-s-indacene (BODIPY) dyes are reported. The use of chromophores or redox active species as \\ $\pi$-spacers, such as BODIPY, requires the inclusion of a sufficiently conjugated donor in order to achieve \\ appropriate charge separation upon photoexcitation. The information derived from this study offers \\ guiding principles for incorporating strongly absorbing, non-innocent $\pi$-spacers in organic dye design.
}

\section{Introduction}

The synthesis of organic compounds capable of facilitating the conversion of solar energy into electrical current has become increasingly popular. ${ }^{1,2}$ Organic dyes for these applications are comprised of a redox-active donor/chromophore (D) that is coupled through a $\pi$-conjugated spacer to an acceptor (A). While thin-film organic photovoltaics typically require aprotic acceptors, dye-sensitized solar cells (DSSCs) require an acceptor capable of anchoring to $\mathrm{TiO}_{2}{ }^{2,3}$ The $\pi$-conjugated linker/bridge is a significant component for manipulating the electronic properties of the dye. For instance, adjusting the length of the conjugated spacer tunes the energy of the frontier molecular orbitals and concomitantly shifts the absorption profile for the desired application. Previous studies involving metal-based dyes ${ }^{4,5}$ have shown that the incorporation of a second redox-active chromophore within the dye architecture can be an effective way to enhance the spectral absorption envelope while facilitating favourable electrontransfer, ultimately leading to improved power-conversion efficiencies.

Boron-dipyrrin dyes have been explored as components in many material applications owing to their tunability and photostability under a wide range of conditions. ${ }^{6}$ Despite this, BODIPY-based dyes have not been effective sensitizers in DSSC applications. ${ }^{7-10}$ Akkaya and coworkers have recently investigated a set of BODIPY dyes possessing Type I and II (Fig. 1)

Department of Chemistry and Biology and the Centre for Urban Energy (CUE), Ryerson University, 350 Victoria St., Toronto, Ontario, Canada, M5B 2 K3.

E-mail: bryan.koivisto@ryerson.ca; Fax: +1-416-979-5044; Tel: +1-416-979-5313 $\dagger$ Electronic supplementary information (ESI) available: Including experimental data and characterization. See DOI: 10.1039/c3ob40213d
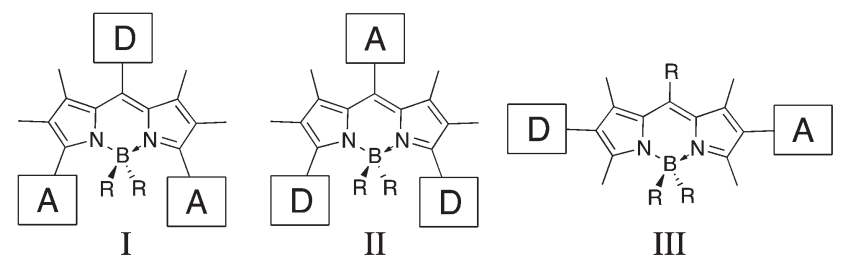

Fig. 1 Type I, II and III donor (D)/acceptor (A) motifs in BODIPY dyes.

D-A motifs. ${ }^{9,10}$ In general, energy transfer through the BODIPY core tends to be less efficient in these types of motifs because substitution to the meso position typically exhibits poor electronic communication; i.e. there is poor conjugation between the BODIPY core. As a result, energy-transfer "cassettes" are observed. ${ }^{6}$ Conversely, extremely fast energy transfer is seen when transition moments are aligned in the Type III architecture. ${ }^{6}$

As a way to further explore the utility of BODIPY as a possible $\pi$-spacer suitable for light-harvesting applications, we have prepared a family of Type III BODIPY dyes modified with a 2,6donor/acceptor motif. Herein, we detail how BODIPYs behave as non-innocent $\pi$-spacers and we examine how subtle changes in conjugation are necessary in order for these dyes candidates to exhibit the appropriate optical and electrochemical properties for light-harvesting.

\section{Synthesis}

Triphenylamine (TPA) was chosen as a donor, owing to its redox robustness. In addition, thiophene spacers were appended to the BODIPY as a means of tuning charge separation between the donor, $\pi$-spacer and acceptor. The synthesis of a family of D-BODIPY-A dyes is presented in Scheme 1. The synthetic protocol presented relies on the 

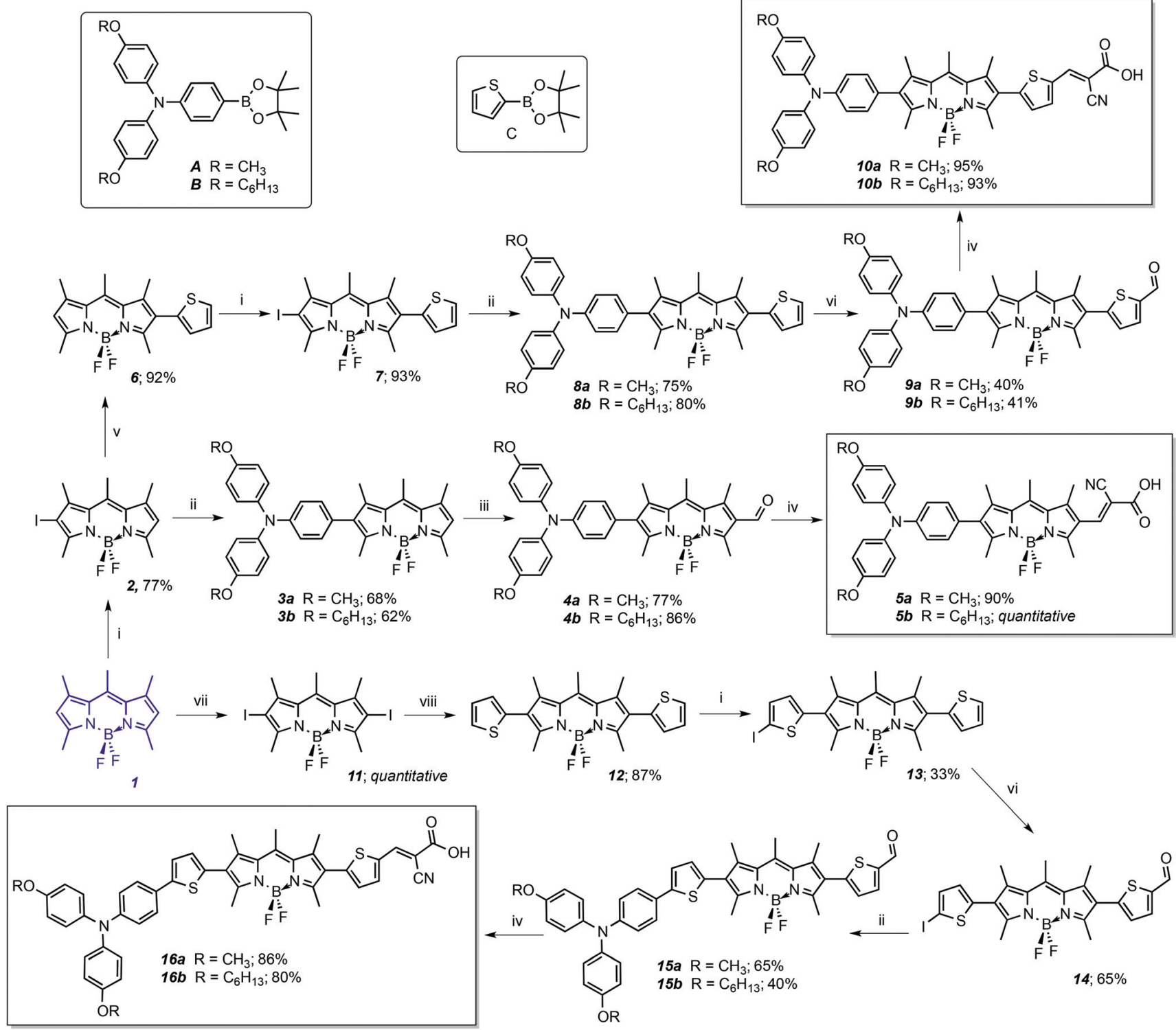

Scheme 1 Synthesis of a family of D-BODIPY-A dyes. Reaction conditions: i: NIS (1 eq.), $\mathrm{CH}_{2} \mathrm{Cl}_{2}, 25^{\circ} \mathrm{C}, 12 \mathrm{~h}$; ii: $\mathbf{A}$ or $\mathbf{B}\left(1.5\right.$ eq.), $\mathrm{K}_{2} \mathrm{CO}_{3}(5 \mathrm{eq}),. \mathrm{Pd}\left(\mathrm{PPh}_{3}\right)_{4}(10 \mathrm{~mol} \%)$, THF- $\mathrm{H}_{2} \mathrm{O} 9: 1$, reflux, 12 h; iii: $\mathrm{POCl}_{3}\left(130\right.$ eq.), DMF (130 eq.), 1,2-dichloroethane, $25{ }^{\circ} \mathrm{C}, 2 \mathrm{~h}$; iv: cyanoacetic acid (2 eq.), piperidine (0.25 eq.), $\mathrm{MeCN}-\mathrm{CHCl}{ }_{3} 1: 1$, reflux, 24 h; v: C (1.5 eq.), $\mathrm{K}_{2} \mathrm{CO}_{3}$ (5 eq.), $\mathrm{Pd}\left(\mathrm{PPh}_{3}\right)_{4}(10 \mathrm{~mol} \%), \mathrm{THF}-\mathrm{H}_{2} \mathrm{O}$ 9: 1, reflux, 12 h; vi: $\mathrm{POCl}_{3}$ (130 eq.), DMF (130 eq.), 1,2-dichloroethane, $25^{\circ} \mathrm{C}, 16$ h; vii: $\mathrm{NIS}$ (2 eq.), $\mathrm{CH}_{2} \mathrm{Cl}_{2}, 25^{\circ} \mathrm{C}, 12 \mathrm{~h}$; viii: $\mathrm{C}\left(3\right.$ eq.), $\mathrm{K}_{2} \mathrm{CO}_{3}$ (5 eq.), $\mathrm{Pd}\left(\mathrm{PPh}_{3}\right)_{4}(10 \mathrm{~mol} \%), \mathrm{THF}-\mathrm{H}_{2} \mathrm{O} 9: 1$, reflux, $12 \mathrm{~h}$.

ability to desymmetrize the BODIPY core, and in most conversions, with the exception of $\mathbf{1 3}$, where substantial di-iodination occurred, good to excellent yields were observed. The Suzuki cross-coupling reaction was employed for all $\mathrm{Ar}-\mathrm{Ar}$ coupling reactions owing to the stability and relatively low toxicity of Suzuki reagents. During the coupling reactions, however, significant decomposition of the BODIPY (base and fluoride sensitive) was observed and as a result, iodide was chosen as the halide to increase the rate of oxidativeaddition. The Vilsmeier-Haack formylation was found to be effective at installing the aldehyde precursor, and Knoevenagel condensations resulted in the desired dyes 5, 10 and 16 in high yields.

\section{Results and discussion}

Electrochemical data in DCM for dyes 5, 10 and 16 and assorted precursors has been presented in Table 1. In all the compounds that contain TPA and BODIPY fragments, three well-resolved and reversible waves were observed by cyclic voltammetry (Fig. 2). These reversible waves include the reduction and oxidation of the BODIPY fragment separated by the oxidation of the TPA moiety. As presented in Table 1, the coupling of a TPA moiety to BODIPY (3a) does very little to the oxidation potential of BODIPY, but stabilizes the LUMO (i.e. the BODIPY reduction potential). In fact, the BODIPY is the better donor, as there is a destabilization in the TPA HOMO (ca. $30 \mathrm{mV})$ 
Table 1 Electrochemical data for dyes $(\mathbf{5}, \mathbf{1 0}, \mathbf{1 6})$ and selected precursors

\begin{tabular}{|c|c|c|c|}
\hline \multirow[b]{2}{*}{ Compound } & \multicolumn{3}{|l|}{$E_{1 / 2}(\mathrm{~V} v s . \mathrm{NHE})^{a}$} \\
\hline & BODIPY/BODIPY ${ }^{\cdot-}$ & $\mathrm{TPA}^{\cdot+} / \mathrm{TPA}$ & BODIPY $^{\cdot+} / \mathrm{BODIPY}$ \\
\hline OMe-TPA $^{b}$ & - & 1.02 & - \\
\hline 1 & -1.05 & - & 1.50 \\
\hline $3 a$ & -0.98 & 0.99 & 1.51 \\
\hline $4 a$ & -0.77 & 1.02 & 1.67 \\
\hline $5 a$ & -0.87 & 1.02 & 1.63 \\
\hline $5 b$ & -0.87 & 1.00 & 1.63 \\
\hline $10 \mathrm{a}$ & -0.90 & 1.01 & 1.58 \\
\hline $10 \mathrm{~b}$ & -0.89 & 0.99 & 1.57 \\
\hline $15 a$ & -0.80 & 0.97 & 1.36 \\
\hline $16 a$ & -0.82 & 0.97 & 1.35 \\
\hline $16 \mathrm{~b}$ & -0.82 & 0.95 & 1.35 \\
\hline
\end{tabular}

${ }^{a}$ Data collected using $0.1 \mathrm{M} \mathrm{NBu}_{4} \mathrm{PF}_{6} \mathrm{DCM}$ solutions at $100 \mathrm{mV} \mathrm{s}^{-1}$ and referenced to a $[\mathrm{Fc}] /[\mathrm{Fc}]^{+}$internal standard followed by conversion to NHE; $[\mathrm{Fc}] /\left[\mathrm{Fc}^{+}\right]=+765 \mathrm{mV} v s$. NHE in DCM. ${ }^{b} \mathrm{OMe}-\mathrm{TPA}=4$-methoxy$N$-(4-methoxyphenyl)- $N$-phenylaniline.

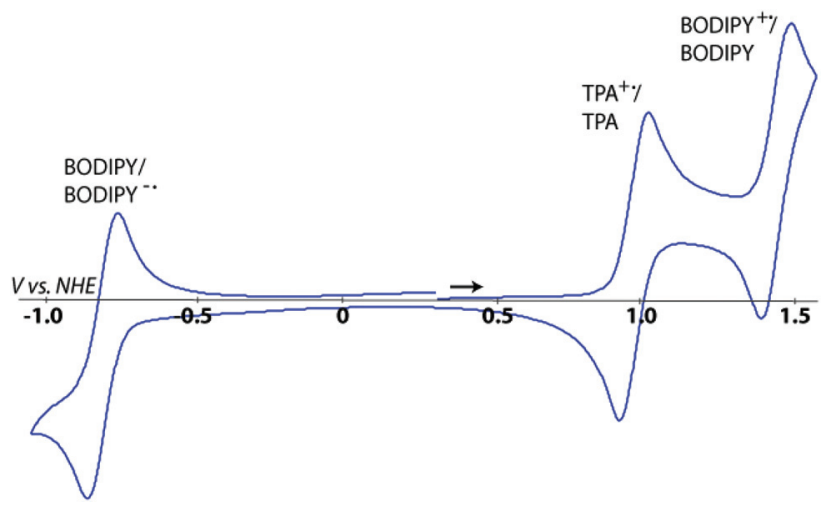

Fig. 2 Cyclic voltammogram of dye $\mathbf{1 6} \mathbf{b}$ vs. NHE in $0.1 \mathrm{M} \mathrm{NBu}_{4} \mathrm{PF}_{6}$ (in $\mathrm{CH}_{2} \mathrm{Cl}_{2}$; $100 \mathrm{mV} \mathrm{s}^{-1}$, Pt working microelectrode).

when compared to bis-OMe-TPA. Installation of the aldehyde (4a) to the BODIPY further stabilizes the LUMO (ca. $200 \mathrm{mV}$ ) and HOMO -1 , as expected for a strong electron withdrawing groups; this effect is diminished when a thiophene spacer separates the withdrawing group from the BODIPY core as exhibited in 15a. Upon condensation of 15a with cyanoacetic acid, the LUMO is destabilized suggesting that the electron withdrawing effect is slightly less for the cyanoacetic acid group. In all the dye families 5, 10, and 16, the TPAs bearing OHex groups are observed to be slightly better donors than $\mathrm{OMe}$ derivatives by $c a .20 \mathrm{mV}$.

The inclusion of thiophene spacers has significant consequences to the electrochemical properties within this dye family. In the absence of a thiophene spacer, the BODIPY oxidation is particularly high owing to the cationic character of the dye upon TPA oxidation. This is true for all dyes in our series but due to the proximity of these redox centres, this effect is most pronounced in $\mathbf{5}$. This can be evidenced by looking at the difference in oxidation potentials between the BODIPY and TPA (BODIPYox-TPAox; 5a $=610 \mathrm{mV} ; \mathbf{1 0}=$ $570 \mathrm{mV} ; \mathbf{1 6}=350 \mathrm{mV}$ ). The true donating effect of TPA is only

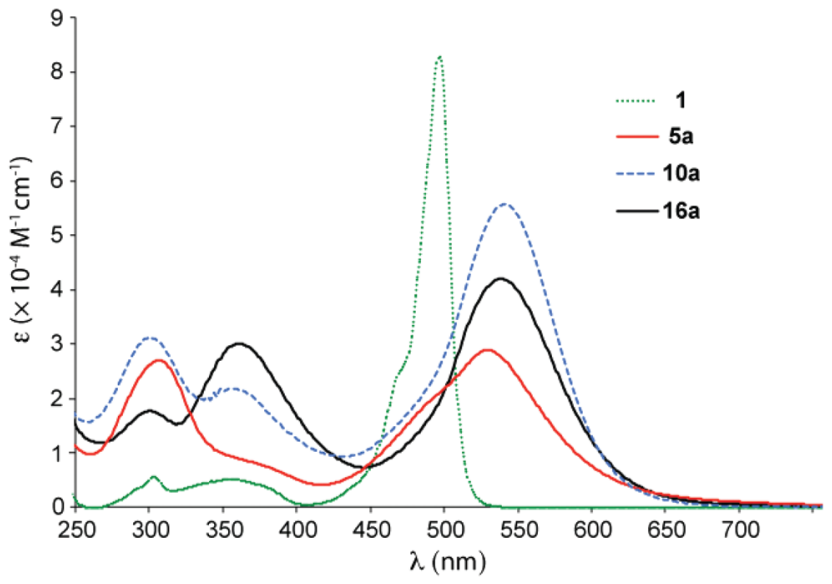

Fig. 3 UV-vis spectra in DCM for BODIPY 1, and -OMe substituted dyes, 5a, $10 a$, and $16 a$.

observed in 16, where the oxidation of BODIPY is lower than that for the parent BODIPY. Presumably, the inclusion of the thiophene permits a more stable planar conformation in the ground state which enhances the resonance donation of the TPA. This delocalization of the HOMO -1 can also be observed from the DFT (B3LYP/6-31G) data presented in Fig. 3.

A representative set of UV-Vis spectra for $\mathbf{1}, \mathbf{5 a}, \mathbf{1 0 a}$, and $16 \mathbf{a}$ are presented in Fig. 3. When compared to BODIPY, 1, it was satisfyingly observed that adding a second redox-active chromophore (TPA) broadened the absorption; however, in contrast to the rich electrochemical behaviour in these systems, altering the conjugated spacer between the two redox-active units has modest effects on the optical properties. The UV-Vis spectra of dyes $\mathbf{5}, \mathbf{1 0}$, and $\mathbf{1 6}$ show three absorptions; two highenergy absorptions at $\sim 300$ and $\sim 350 \mathrm{~nm}$ and one broad low energy transition centred $c a$. at $550 \mathrm{~nm}$. From TD-DFT (B3LYP/ 6-31G) the high-energy absorptions are assigned as HOMO - 1 to LUMO + 1 and LUMO + 2 transitions, and increasing the conjugation around the BODIPY core, via thiophene substitution, results in the increased extinction coefficients at $c a$. $350 \mathrm{~nm}$. Additionally, all the dyes possess a broad absorption centred around $c a .550 \mathrm{~nm}(450-650 \mathrm{~nm})$, which is assigned as the HOMO - 1 to LUMO transition; this band red-shifts slightly as the conjugation is extended via thiophene inclusion.

The dominant transition in the absorption spectra of all dyes, calculated by TD-DFT, is that of the HOMO - 1 to LUMO. Unfortunately, as observed in Fig. 4, this transition in $\mathbf{5}$ and $\mathbf{1 0}$ does not favourably perturb TPA electron density toward the acceptor in the excited state. However, the extended delocalization in the HOMO - 1 orbital of $\mathbf{1 6}$ suggests that the electronic transition leading to absorption in this family could redistribute the electron density appropriately toward the acceptor/ anchor. This is also consistent with the electrochemical studies in solution where the oxidation potential of BODIPY is lowered due to the presence of the thiophene-TPA fragment.

For light-harvesting applications, excited state electron density needs to be localized near the acceptor. As seen in this 


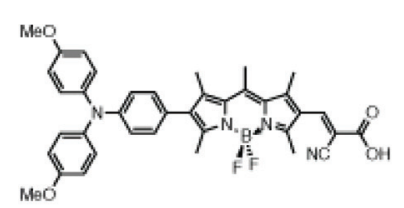

$5 a$

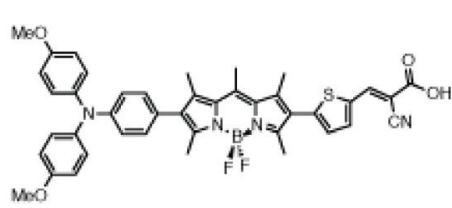

$10 a$

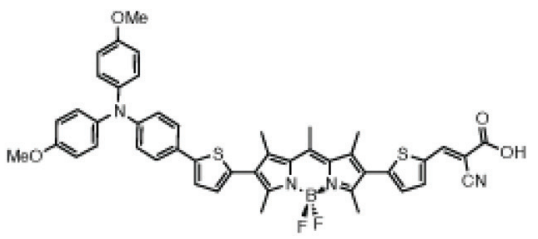

$16 a$
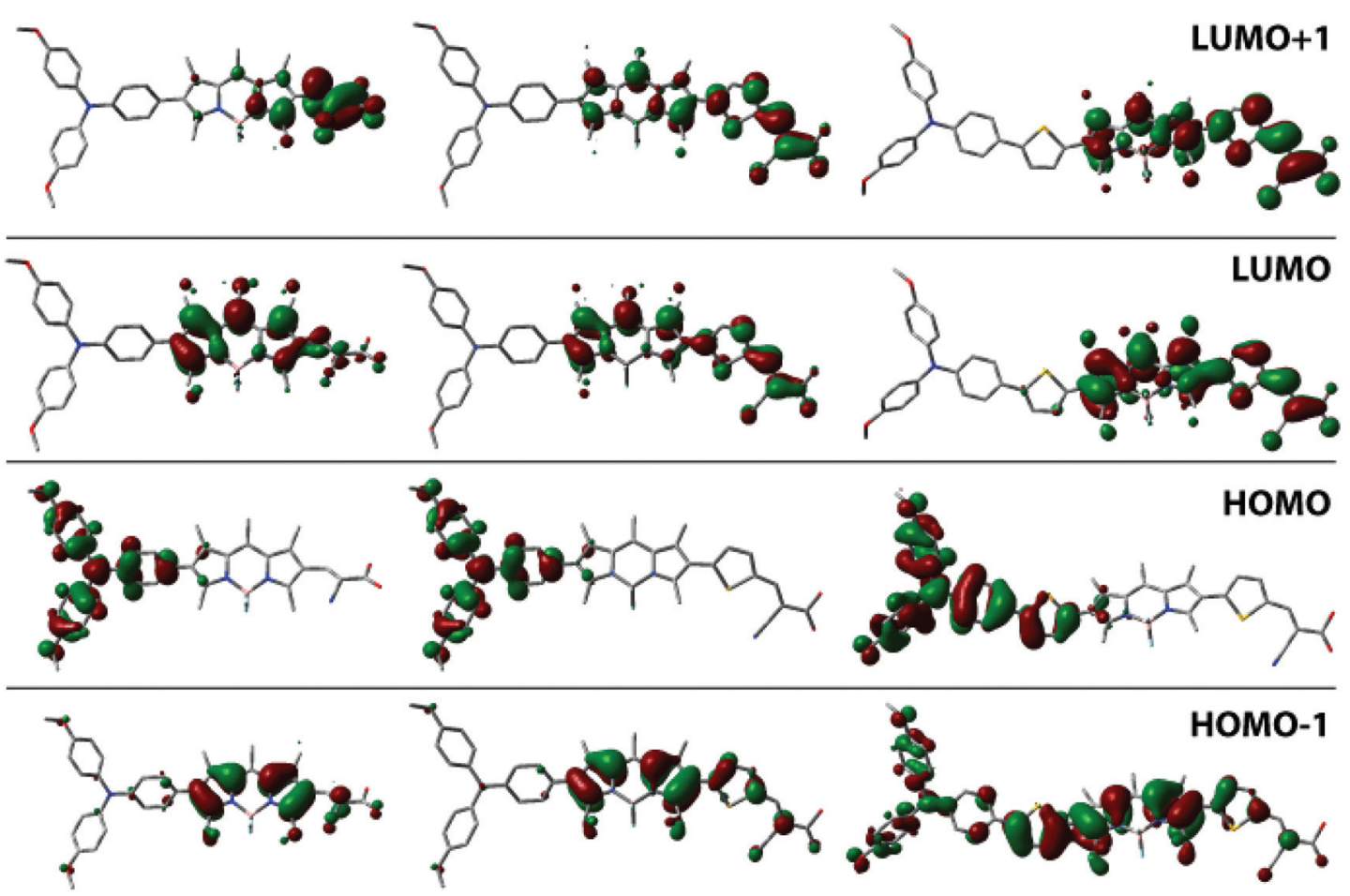

Fig. 4 Frontier molecular orbitals as predicted by DFT (B3LYP/6-31G) for dyes 5a, 10a, 16a

family of BODIPY dyes, the presence of non-innocent $\pi$-spacers poses a challenge for light-harvesting, because the dominant optical transition is so strongly confined to the BODIPY portion of the dye. In order to make use of this strong optical transition, it is essential that significant charge separation can occur in the excited state. As demonstrated here, conjugation between the TPA donor and the non-innocent $\pi$-spacer is a necessary requirement for charge separation in the excited state. While Type III 2,6-substituted BODIPYs are promising dye candidates because of their intense absorption, this work demonstrates that appropriate excited state electronic redistributions are only achieved by thoughtful dye design, using strong donors that are effectively conjugated (i.e., using thiophenes) to the non-innocent $\pi$-spacer.

\section{Conclusions}

It is unlikely, owing to poor charge separation in the dominant optical transition, that dye families $\mathbf{5}$ or $\mathbf{1 0}$ would be effective sensitizers in DSSC applications despite having the appropriate LUMO energies for injection; this is also consistent with previous observations. $^{7,8}$ However, preliminary DSSC device data (Table S2†) suggests that family $\mathbf{1 6}$ is a much better dye owing to the dominant optical transition involving the greatest charge separation towards the anchor. Extensive DSSC devices studies are currently underway and this will be disseminated in a future study.

\section{Acknowledgements}

This work was financially supported by the Natural Science and Engineering Research Council (NSERC) of Canada, the Centre for Urban Energy (CUE) and Ryerson University.

\section{Notes and references}

1 R. K. Kanaparthi, J. Kandhadi and L. Giribabu, Tetrahedron, 2012, 68, 8383-8393.

2 A. Mishra, M. K. R. Fischer and P. Bauerle, Angew. Chem., Int. Ed., 2009, 48, 2474-2499.

3 A. Hagfeldt, G. Boschloo, L. Sun, L. Kloo and H. Pettersson, Chem. Rev., 2010, 110, 6595-6663. 
4 K. Hu, K. C. D. Robson, P. G. Johansson, C. P. Berlinguette and G. J. Meyer, J. Am. Chem. Soc., 2012, 134, 8352-8355.

5 K. C. D. Robson, B. Sporinova, B. D. Koivisto, E. Schott, D. G. Brown and C. P. Berlinguette, Inorg. Chem., 2011, 50, 6019-6028.

6 A. Loudet and K. Burgess, Chem. Rev., 2007, 107, 4891-4932.

7 J.-B. Wang, X.-Q. Fang, X. Pan, S.-Y. Dai and Q.-H. Song, Chem.-Asian J., 2012, 7, 696-700.
8 M. Mao, J.-B. Wang, Z.-F. Xiao, S.-Y. Dai and Q.-H. Song, Dyes Pigm., 2012, 94, 224-232.

9 S. Kolemen, O. A. Bozdemir, Y. Cakmak, G. Barin, S. ErtenEla, M. Marszalek, J.-H. Yum, S. M. Zakeeruddin, M. K. Nazeeruddin, M. Graetzel and E. U. Akkaya, Chem. Sci., 2011, 2, 949-954.

10 S. Erten-Ela, M. D. Yilmaz, B. Icli, Y. Dede, S. Icli and E. U. Akkaya, Org. Lett., 2008, 10, 3299-3302. 